0 ince the atomic bombings of Hiroshima and Nagasaki in 1945, and the subsequent reports of raised cancer risks among survivors in the two cities, there has been interest and concern about risks arising from this and other nuclear incidents, such as the 1986 Chernobyl accident. This article reviews methodological aspects associated with the detection of cancer risks in groups exposed to ionising radiation, summarises findings from relevant studies, and looks at possible future developments.

It should be emphasised that cancer risks have also been examined in a multitude of studies of radiation exposures from medical, occupational, and natural sources. Further details of these studies can be found in the most recent report by the United Nations Scientific Committee on the Effects of Atomic Radiation (UNSCEAR). ${ }^{1}$

\title{
METHODOLOGICAL ASPECTS
}

The ability to detect and quantify raised cancer risks in an epidemiological study depends on the study design. Particular aspects are as follows.

\section{Statistical power}

Owing to random variation in data, it may not be possible to identify a raised risk in a small study. Therefore, before conducting a study, it is standard practice to calculate its statistical power. This is the probability that the study will detect a given level of raised risk with a specific degree of confidence-for example, the probability of detecting a doubling of risk using a significance test at the $5 \%$ level. For a cohort study, in which a cohort of individuals is followed to determine their subsequent disease incidence or mortality, the power depends on the size of the cohort, the length of time for which they are followed up, the baseline rate of cancer, the distribution of radiation exposures received by members of the cohort, and the putative radiation risk factor. For a case-control study, in which radiation exposures are compared for persons with the cancer of interest (cases) and persons selected from the same source population who do not have this cancer (controls), the power depends on the numbers of cases and controls, the frequency and level of exposure, and the putative radiation risk factor. Power calculations should not be necessary once a study has been conducted, since the precision of the study can be gauged by the width of the confidence interval for the estimated radiation effect.

In situations where radiation doses are all very low or if the range of doses is very narrow, then the power to detect an effect is usually small. This applies to many nuclear incidents, as indicated below.

\section{Bias and confounding}

In any epidemiological study, it is important to minimise the potential for systematic errors (bias) or misleading findings due to a variable that it is correlated with both the disease and exposure under study (confounding). Bias or confounding can arise in various ways. For example, bias might occur in a prospective study from systematic differences in the ascertainment of cancer between persons with differing levels of exposure, or in a retrospective study by selecting case and control subjects from somewhat different populations, perhaps because of low participation rates. For a disease with strong risk factors (for example, lung cancer and smoking), failure to adjust for potential confounding factors can have a large impact on the study findings, particularly when trying to detect what might be a small radiation effect. These problems are usually greater in correlation (sometimes called ecological) studies based on aggregated data-for example, disease rates in different areas and possibly at different times-than in cohort or case-control studies. This is because of the so-called "ecological fallacy", under which the results from analysis of aggregated data on disease risk and exposures can differ artefactually from the analysis of data at the individual level. Even adjusting for aggregated data on confounders such as smoking may not

Correspondence to: Dr Colin Muirhead, National Radiological Protection Board, Chilton, Didcot, Oxon, OX11 0RQ, UK

colin.muirhead@nrpb.org.uk be sufficient to adjust for the biases that might arise in correlation studies. Consequently, while correlation studies can sometimes be useful for monitoring disease rates, more substantial inferences should be based on cohort or case-control studies. Further details of the advantages and disadvantages of different types of epidemiological study are given in standard epidemiological textbooks (for example, MacMahon and Trichopoulos ${ }^{2}$ ). 


\section{Exposure assessment}

A key aspect in estimating cancer risks following radiation exposure relates to the assessment of radiation doses. It is possible sometimes to reconstruct doses on an individual basis-for example, using measurement data and/or obtaining information from the study subjects. Such approaches have been used for the Japanese atomic bomb (A-bomb) survivors and some groups of persons exposed to fallout from the Chernobyl accident, as described below. However, difficulties in obtaining suitable measurements or in recalling past events can give rise to uncertainties in these dose estimates.

Provided that doses are assessed independently of whether the study subjects develop cancer, there will not be bias owing to differential misclassification of exposures, as, for example, would arise from selective recall by the subjects of past exposures. However, non-differential misclassification can still lead to bias in estimating dose-response relationships. In particular, random errors in individual dose estimates tend to bias any trend in risk with dose towards the null. It is possible to adjust for this bias using statistical techniques. However, such dosimetric errors can decrease the statistical power of a study, particularly when the predicted raised level of risk is low.

Biological and physical methods of dosimetry are now being incorporated into some epidemiological studies. For example, the glycophorin A mutational assay of red blood cells and the FISH (fluorescent in situ hybridisation) technique for chromosome stable translocation analysis have been used in investigations of Chernobyl clean-up workers, and electron spin resonance of tooth enamel has been used in A-bomb survivors in Japan. ${ }^{1}$ However:

- it is generally difficult to evaluate individual doses of $0.1-$ 0.2 sievert $(\mathrm{Sv})$ using these methods;

- it can be difficult and/or expensive to collect, store, and analyse material for large numbers of people;

- some biological measures can be affected by factors other than radiation (for example, age and smoking can influence chromosome translocation frequencies);

- the effect of radiation on some biological measures, such as dicentric aberrations, is relatively short lived, so the collection of related materials is unlikely to be useful in studying exposures received many years previously.

In some instances it is not possible to estimate doses or surrogates for exposure on an individual basis, so aggregated values have been used. For example, some studies have looked at groups resident in different areas or at different times, as a means of assigning exposures. This approach, which is used in correlation studies, is less sound methodologically than obtaining dose estimates on an individual basis, certainly for the purposes of risk estimation. ${ }^{1}$

\section{Conclusions on methodology}

- Not all epidemiological studies of nuclear incidents are informative about cancer risks from radiation exposures.

- It is difficult generally to draw conclusions from studies either that involve only very low radiation doses, that are based on a small number of cancer cases, or that may be subject to substantial bias or confounding.

- In contrast, well designed and conducted studies with large numbers of cases and a wide range of doses, preferably identified on an individual basis, should provide more reliable results.

The above criteria will be used in evaluating the following studies.

\section{Review of studies}

Nuclear weapons

Hiroshima and Nagasaki

The Life Span Study (LSS) of the atomic bombings of Japan in 1945 has many of the desirable qualities for an epidemiological study of the effects of radiation exposure. The most recent findings on mortality, ${ }^{3}$ covering the period 1950 to 1990 , are based on a cohort of over 86000 survivors in the two cities, who received radiation doses ranging from essentially zero up to several $\mathrm{Sv}$ - persons with higher doses mostly died within a few weeks of the explosions or from the direct effects of the explosions (blast and heat). The doses were mainly from external exposures-mostly from $\gamma$ radiation-received at the time of the explosions. Information on individuals' location and degree of shielding at the time of the bombings has been utilised together with estimates of the weapon yield and calculations of the transport of radiation through the air in order to estimate doses on an individual basis. However, there are still some uncertainties in parts of the dose calculations-specifically for neutron doses in Hiroshima, which may have been underestimated. The cohort contains persons of all ages and both sexes, with an essentially complete mortality follow up within Japan. Information on cancer incidence has also been collected in the two cities. ${ }^{45}$ The large cohort and long term follow up means that the study has high statistical power overall, although the power is less when examining some of the rarer cancers.

As of the end of $1990,44 \%$ of the LSS population had died, of whom 7827 had died from cancer. ${ }^{3}$ Based on analyses of cancer risks in relation to dose, Pierce et al estimated that around 87 of the leukaemia deaths and 334 of the deaths from solid cancers could be attributed to radiation exposure. ${ }^{3}$ The radiation induced leukaemia risk appears to have been largely expressed during the follow up period, and the lifetime excess absolute risk of leukaemia associated with an acute dose of $1 \mathrm{~Sv}$ has been estimated by UNSCEAR ${ }^{1}$ as being about 1 in 100. However, in contrast to leukaemia, nearly a quarter of the radiation induced solid cancers are estimated to have arisen in the most recent five year period of the mortality follow up - that is, 1986-90. ${ }^{3}$ Since most of the A-bomb survivors exposed at young ages are still alive, the future pattern of cancer risks in this group will be important in determining lifetime risks. Assuming that the radiation induced risk will continue to vary in proportion to the baseline risk of solid cancers, UNSCEAR ${ }^{1}$ has estimated the lifetime risk of solid cancer mortality following an acute dose of $1 \mathrm{~Sv}$ to a population of all ages to be about 11 in 100. This model predicts that lifetime risks will be higher for exposure in childhood than in adulthood, reflecting the difference by age in the relative risks observed to date. However, if the relative risks were to decrease in future, then the lifetime risks may not differ greatly by age at exposure, and the lifetime risk averaged over all ages may be about 30\% less than the above value. ${ }^{1}$

Figure 1 shows how cancer risks vary by dose. Overall, risks for solid cancers tend to be consistent with a linear dose-response relationship below about $3 \mathrm{~Sv}^{35}$ Among individual types of solid cancer, only for non-melanoma skin cancer incidence is there a suggestion of non-linearity. For leukaemia, a linear quadratic model — such that the risk per unit dose is smaller at low rather than high doses-provides a significantly better fit than a linear model to data below about $3 \mathrm{~Sv}$ on both incidence ${ }^{4}$ and mortality ${ }^{3}$ in the LSS. In particular, Pierce et al estimated the radiation induced leukaemia risk at $0.1 \mathrm{~Sv}$ to be only about half that expected 


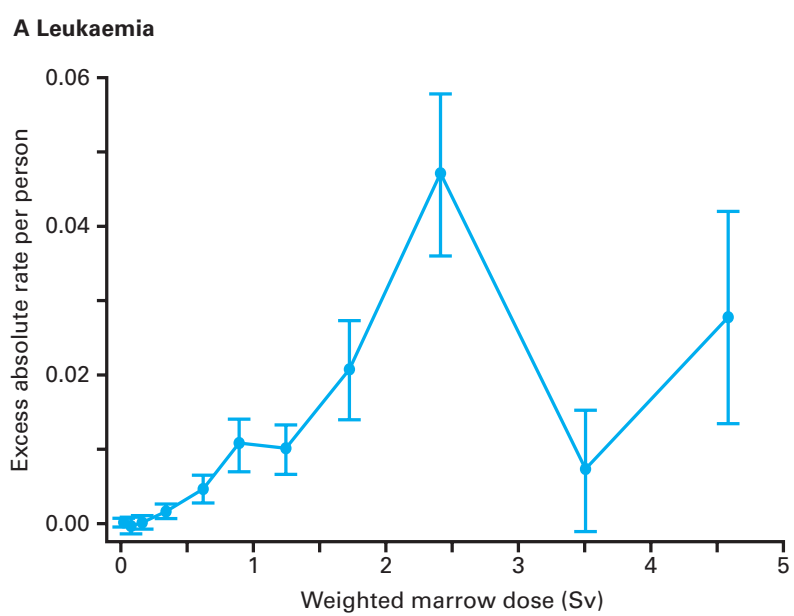

B All solid cancers

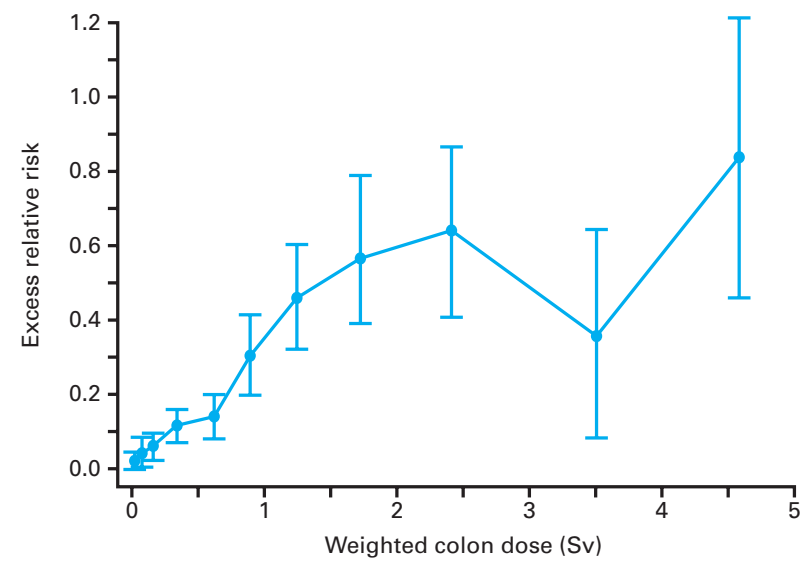

Figure 1 Variation in cancer mortality rates by radiation dose among survivors of the atomic bombings in Fapan. Reproduced from Pierce et al with permission of the publisher.

by linear extrapolation from the risk at $1 \mathrm{~Sv}^{3}$ This non-linear model for leukaemia and a linear model for solid cancers were recommended by UNSCEAR ${ }^{1}$ as a means of estimating risks from acute doses below $1 \mathrm{~Sv}$, based on the risk coefficients at $1 \mathrm{~Sv}$ that are cited above. The LSS does not provide information on chronic radiation exposures; data on this topic from other sources have been reviewed by UNSCEAR. ${ }^{6}$

Figure 2 shows estimates of the excess relative risk (ERR) per Sv for various types of solid cancer, adjusted for age at exposure and sex, based on mortality in the LSS. ${ }^{3}$ The ERR equals the relative risk minus one. While the variation between cancer sites in the ERR per Sv is not significant, Pierce et al noted that this measure of risk may be expected to vary owing to differences in the aetiologies of the various cancer types. ${ }^{3}$ Although not presented here, the excess absolute risk per $\mathrm{Sv}$ would show greater variation between cancer sites than the ERR per Sv, once account is taken of differences in baseline rates between these cancer types.

\section{Weapons testing}

Various studies have examined cancer risks in groups exposed to fallout from atmospheric nuclear weapons testing. In contrast to the Japanese A-bomb survivors, these groups were generally many miles from the hypocentres of the explosions, and so their radiation exposures often arose from intakes of radionuclides (for example, radioiodine), as well as external $\gamma$ radiation in some instances. Another contrast with

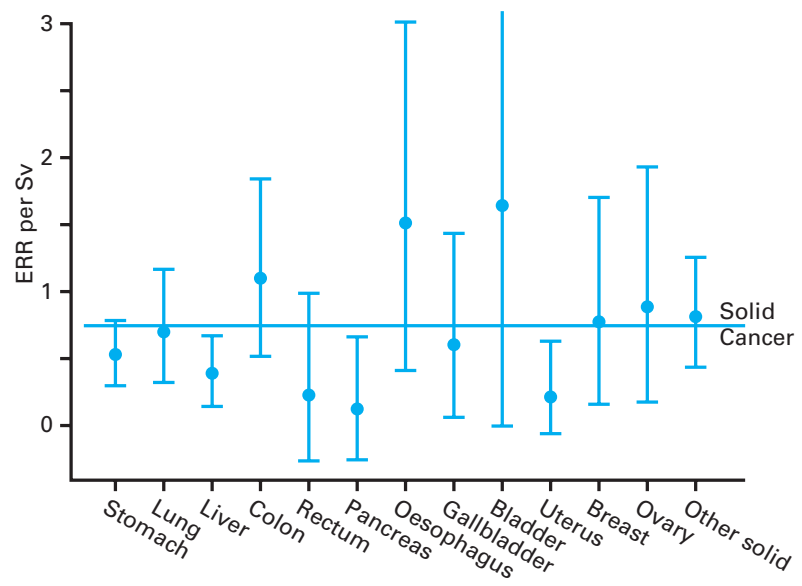

Figure 2 Excess relative risk for mortality (and 90\% confidence intervals) from specific solid cancers and all solid cancers combined (horizontal line) among survivors of the atomic bombings in fapan, standardised for women exposed at age 30 years. Reproduced from Pierce et al $l^{3}$ with permission of the publisher.

Box 1: Japanese A-bomb survivors
- Life Span Study provides information on long term risks
from acute exposure over a wide range of doses, for a
population of all ages and both sexes
- Increased risks identified for leukaemia and many types of
solid cancer
- Variation in radiation risks with dose, age, and time has
been quantified, although there are some uncertainties
- Continued follow up important in improving estimates of
lifetime cancer risks

the Japanese A-bomb study is that most of these studies of weapons fallout were either correlation in design and/or lacked information on individual exposures.

Particular attention has been directed in the fallout studies towards cancer of the thyroid, which has been shown from the Japanese studies and from studies of medical irradiation to be particularly radiosensitive following exposure in childhood. ${ }^{17}$ There appeared to be a raised prevalence of thyroid cancer among inhabitants of the Marshall Islands in the Pacific, who received high radiation doses (of the order of several sievert or more) to the thyroid in childhood from fallout from a US test in 1954, although the number of cases was fairly small. ${ }^{7}$ Rates of thyroid cancer in French Polynesia were higher than those in reference groups in Hawaii and New Zealand; however, this raised risk was similar for those Polynesians potentially exposed in childhood to fallout from the French atmospheric tests conducted between 1966 and 1974 and for those born earlier. ${ }^{8}$ In a cohort of persons exposed to fallout from weapons tests conducted at the Nevada test site in the USA in the 1950s, there was some indication of an association between thyroid cancer and exposure to ${ }^{131} \mathrm{I}$ in childhood, although the number of cases was small. ${ }^{9}$ A national geographical and temporal correlation study in the USA reported a raised risk of thyroid cancer in relation to estimated dose from ${ }^{131} \mathrm{I}$ received in the first year of life from weapons fallout, but no association was found for exposure at later ages ${ }^{10}$; inferences are limited by the lack of individual data.

Leukaemia has also been the focus of investigation, again because of other studies that have demonstrated its radiosensitivity. ${ }^{1}$ A case-control study in southwestern Utah in the USA found an association between bone marrow doses of up to around $0.3 \mathrm{~Sv}$ (mostly from $\gamma$ radiation) 
caused by testing at the Nevada test site and leukaemia mortality, mainly for exposure in childhood or adolescence ${ }^{11}$; the estimate of the risk per unit dose was uncertain, although it was consistent with values derived from the Japanese A-bomb survivors. In a cohort of over 200000 US military personnel who participated in the US weapons test programme, cancer mortality was generally similar to that in a reference group, with the exception of leukaemia, which was raised among participants at the Nevada test site. ${ }^{12}$ Results from some other studies of nuclear weapons test participants are summarised by UNSCEAR in its 1994 report. ${ }^{13}$ A correlation study in the Nordic countries suggested a raised risk of childhood leukaemia in connection with the period when global fallout from weapons testing in the 1950 s and 1960 s was at its highest. ${ }^{14}$ The estimated risk coefficient from this study is consistent with that from the Japanese A-bomb survivors, although precise quantification of risks is difficult in view of the low doses (generally $<0.002 \mathrm{~Sv}$ on average) and the correlation nature of the study.

Studies are in progress of groups of about 10000 people in Kazakhstan and 40000 people in the Altai region of Russia who received average doses of over $0.25 \mathrm{~Sv}$ as a result of fallout from some of the atmospheric weapons tests conducted at the Semipalatinsk test site between 1949 and 1962. ${ }^{1}$ The follow up of cohorts and estimation of doses are challenging tasks, given the many years that passed between the tests and the start of the epidemiological programmes in these areas. $^{715}$

\section{Incidents at nuclear plants \\ Chernobyl}

The most serious accident in the history of the nuclear industry took place at the Chernobyl nuclear power plant in the Ukraine on 26 April 1986. It led to the deaths of 30 power plant employees and firemen within a few weeks, mostly from acute radiation effects, and to the evacuation of over 100000 people from surrounding areas. Large parts of Belarus and Ukraine, plus parts of the Russian Federation, were contaminated and approximately five million people still live in areas of these countries with levels of ${ }^{137} \mathrm{Cs}$ ground deposition of more than $37 \mathrm{kBq} / \mathrm{m}^{2}$. Other countries in the northern hemisphere-principally in northern and eastern Europe-were also affected by radioactive releases from the plant, although generally to a much lower extent than in the former Soviet Union. ${ }^{1}$

The most notable finding from health studies following the Chernobyl accident has been the excess of thyroid cancer among those exposed in childhood in the severely contaminated areas of Belarus, Ukraine, and the Russian Federation. ${ }^{17}$ About 1800 cases were reported during 1990 to 1998, with the risk appearing to be greatest for those exposed at very young ages. ${ }^{1}$ Questions were raised initially about whether these findings might reflect the effect of screening programmes. However, while some tumours may have been diagnosed earlier than would have otherwise been the case, most of the tumours diagnosed were aggressive rather than being occult. Almost all of the epidemiological studies conducted to date on this topic have been correlation studies, with analyses being performed on geographical and temporal variation of thyroid cancer rates ${ }^{17}$; as noted earlier, there are limitations to this approach. An exception has been a case-control study conducted in Belarus which-in common with the correlation studies-has shown an association between thyroid cancer risk and estimated thyroid dose from ${ }^{131} \mathrm{I} .{ }^{16}$ However, the quantification of risks is difficult because:
- estimates of radiation doses to the thyroid are uncertain, partly because of the possible contribution of short term radioiodines;

- many of the regions are iodine deficient and iodine dietary supplementation had been stopped before the accident, so possibly affecting risks;

- other factors such as genetic susceptibility might modify risks from radiation.

Research on these issues is continuing. ${ }^{75}$ It will also be important to continue to monitor those exposed at young ages for many years into the future, in order to evaluate the long term risks.

In contrast to thyroid cancer, correlation studies in the affected areas of the former Soviet Union have not demonstrated raised risks of other cancers, such as childhood leukaemia. ${ }^{1}$ A pan-European correlation study $^{17}$ indicated a slight increase in childhood leukaemia incidence following the accident, but this was not related to the geographical variation in doses, which were low (generally $<0.001 \mathrm{~Sv}$ ) in most of the regions studied. A raised risk of infant leukaemia has been reported in Greece following the accident, ${ }^{18}$ but studies in Belarus and Germany have not shown links between Chernobyl exposures and infant leukaemia. ${ }^{1}$

Several cohort and case-control studies have been conducted of workers who took part in the clean up of Chernobyl following the accident. There are methodological problems with some of the studies that have reported findings to date, reflecting small numbers, the lack of an adequate comparison group, and/or uncertainties in dose estimates. The more reliable of these studies have not demonstrated an association between risk of leukaemia or other cancers and radiation exposure at Chernobyl. ${ }^{1}$ However, further studies are in progress that may provide more definitive information on risks among these workers. ${ }^{15}$ As mentioned earlier, a particular challenge is to obtain good individual dose estimates, in view of concerns as to the accuracy of the workers' official doses.

Box 2: Chernobyl
- Large areas of Belarus, Ukraine, and western Russia were
contaminated following the 1986 accident
Substantial increase in thyroid cancer following childhood
exposure was found in these areas
Other types of cancer have not yet been shown to be
increased
Long term, well designed studies are necessary to provide
further information on cancer risks

\section{Other incidents}

This section refers only to nuclear plants where incidents are known to have occurred. Studies of cancer around nuclear installations generally have been reviewed by Muirhead. ${ }^{19}$

Doses to both persons living near sites of nuclear incidents and workers at these plants have generally been lower than those associated with the Chernobyl accident. For example, doses to people within a few miles of the Three Mile Island nuclear plant in the USA at the time of the 1979 accident were $0.25 \mathrm{mSv}(0.00025 \mathrm{~Sv})$ or less on average - that is, less than a year's exposure to natural background radiation. Neither an early correlation study ${ }^{20}$ nor a later cohort study ${ }^{21}$ provided consistent evidence of an association between cancer rates around this plant and radiation releases. Workers at Sellafield in the UK involved in the 1957 Windscale reactor fire generally received doses of $<0.015 \mathrm{~Sv}$ as a consequence; cancer rates in this group were not raised, 
although the statistical power was low owing to the small cohort (470 workers). ${ }^{22}$ While it has been estimated from a dose assessment that several tens of people throughout the UK might have died as a result of radiation releases from the Windscale accident, ${ }^{23}$ this has not been verified epidemiologically; in particular, it would be difficult to detect such an excess among a population of tens of millions of people followed for several decades.

Between 1944 and 1957, the Hanford nuclear site in the USA released large quantities of radioactive iodine into the atmosphere. A study has been conducted of over 3000 people born in surrounding areas during 1940-46, among whom any effects might be expected to be concentrated. ${ }^{24}$ Individual doses were estimated in an environmental dose reconstruction project, using information on factors such as level of milk consumption. Thyroid doses were highly skewed, with a mean of just under $0.2 \mathrm{~Sv}$ and some values above $2 \mathrm{~Sv}$. No association was found between either thyroid cancer or benign thyroid nodules-identified in clinical examinations of cohort members-and radiation dose, although these doses were uncertain. ${ }^{24}$

The production of plutonium in Russia started at the Mayak nuclear site in the southern Urals in 1949. Studies of workers at Mayak have been reviewed by UNSCEAR. ${ }^{1}$ During the early years of operation, large amounts of radioactive materials were discharged directly into the nearby Techa River, both routinely and owing to leaks. People who lived near the river during the 1950s received doses of radiation - both externally and internally (for example, from strontium and plutonium) - that ranged from low values up to more than $1 \mathrm{~Sv}$, averaged over the body. Some of these people were also exposed following the explosion of a high level radioactive waste container at Mayak in 1957 (known as the Kyshtym accident), which contaminated parts of the eastern and southern Urals. Since the late 1960s, systematic follow up has been conducted of a fixed cohort of about 26500 people who lived near the Techa River during 1949-52, when releases from Mayak were highest. The large number of people exposed to a reasonably wide range of doses suggests that the Techa River population may potentially be valuable for radiation risk assessment, particularly for protracted and internal exposures. However, as of 1990, the vital status was unknown for about a third of the cohort members who were not known to have left the region, and the cause of death was unknown for $30 \%$ of those known to have died. ${ }^{25}$ Considerable effort has been directed to estimating doses from external and internal sources, in part using biological techniques, but there are uncertainties in individual estimates. Nevertheless, in common with the Japanese A-bomb survivors, the Techa River cohort covers a population of all ages and both sexes, exposed to a wide range of doses, and followed up for many years. Furthermore, whereas the Japanese cohort essentially received an instantaneous external dose, the Techa River cohort received doses both externally and internally over a number of years. A preliminary comparison of the two cohorts indicates that both studies show increases in leukaemia with increasing dose. ${ }^{25}$ Further work is in progress to improve both the dose assessment and the follow up for the Techa River study, which will be important in order to draw more definitive conclusions.

\section{Conclusions}

While many studies have been conducted of cancer following nuclear incidents, only a few of them have been sufficiently strong methodologically to provide useful information on

\section{Box 3: Key conclusions}

- Not all studies of cancer following nuclear incidents are informative; attention needs to be paid to the potential for bias or confounding, and to the level of statistical power

- The Life Span Study of the Japanese A-bomb survivors has provided substantial information on how risks from an acute radiation dose vary by dose, age, time, and cancer type. More information will be gained by continued follow up

- Studies of incidents in the former Soviet Union may provide additional information on radiation induced cancer, particularly for protracted and internal exposures, but there are difficulties in performing cohort and case control studies in this region

- Many of the studies conducted in other countries are unsuitable for calculating radiation risks, although some of these studies do allow a rough check to be made of existing risk estimates

radiation induced cancer risks. The Life Span Study of the Japanese A-bomb survivors has shown how the risks of various types of cancer vary according to radiation dose following an instantaneous external exposure, and how these risks may differ by factors such as age at exposure and time since exposure. Further follow up of this cohort will be valuable in obtaining more precise information on the role of these factors, including the magnitude of cancer risks over a full lifetime.

Among the other studies considered here, those of populations exposed in the former Soviet Union have the most potential to provide additional information on cancer risks, particularly concerning the effects of protracted external or internal exposures. In contrast to many other studies, large numbers of people were exposed to a wide range of doses as a consequence of either the Chernobyl accident, living near the Techa River, or fallout from the Semipalatinsk test site. Continued investigation of thyroid cancer rates among persons exposed in early life to Chernobyl fallout will be particularly important, to see whether these rates continue at their current high level. It should be emphasised that performing large cohort or casecontrol studies in the former Soviet Union is not always easy, owing to problems with, for example, the identification of cohorts many years after exposure, loss of persons to follow up, and uncertainties in dose estimation. Nevertheless, steps are being taken to improve studies in these areas, with the aim of providing more useful information in the future.

Outside Japan and the former Soviet Union, several of the studies cited here have suggested raised risks of thyroid cancer or leukaemia, while others have not. Many of these studies are unsuitable for assessing radiation risks, given problems of low statistical power and the potential for bias or

\section{Box 4: Important aspects of future studies}

- Need good study design-preferably cohort or casecontrol-and prior assessment of statistical power

- Minimise sources of bias-for example, in selecting subjects or in collecting cancer data

- Attempt to obtain radiation dose estimates that are as accurate and precise as possible, or at least be able to quantify the uncertainties in these estimates

- Obtain information on potentially important confounding variables

- Analyse using appropriate statistical methods 
confounding, although some of them-mainly those that collected data at the individual level - do provide a rough check on existing estimates.

With regard to cancer prevention, the best situation would be to ensure that significant accidents do not occur. However, given the possibility that accidents might arise, it is important when formulating emergency plans to use the best available information on radiation risks - not only from the studies considered here but also from other types of studies, ${ }^{1}$ as mentioned earlier. Furthermore, accident response planning needs to balance radiation risks against the potential detriment associated with countermeasures, as practised in the UK. ${ }^{26}$ Finally, when considering whether to conduct an epidemiological study following an incident, it is important to examine whether it could provide useful information and, if so, to ensure that the study is then performed well.

The author would like to thank Don Pierce, Dale Preston, and the journal Radiation Research for their permission to reproduce the figures. ${ }^{3}$ Thanks also to Gerry Kendall and Mary Morrey for their comments on an earlier version of this paper.

\section{References}

1 United National Scientific Committee on the Effects of Atomic Radiation (UNSCEAR). Sources and effects of ionizing radiation. 2000 Report to the General Assembly, with scientific annexes. New York: United Nations, 2000.

- This report provides a comprehensive overview of studies on cancer risk following radiation exposure, both from nuclear incidents and from other sources.

2 MacMahon B, Trichopoulos D. Epidemiology, principles and methods, 2nd ed. Boston: Little, Brown and Co, 1996.

- An understanding of the strengths and weaknesses of epidemiology-as outlined in this and other standard textbooks-is key to the evaluation of studies.

3 Pierce DA, Shimizu Y, Preston DL, et al. Studies of the mortality of A-bomb survivors. Report 12, part 1. Cancer: 1950-1990. Radiat Res 1996;146:1-27.

- This describes the most recent mortality follow up of the Japanese A-bomb survivors.

4 Preston DL, Kusumi S, Tomonaga M, et al. Cancer incidence in atomic bomb survivors. Part III: leukemia, lymphoma and multiple myeloma, 1950-1987. Radiat Res 1994;137:S68-97.

5 Thompson DE, Mabuchi K, Ron E, et al. Cancer incidence in atomic bomb survivors. Part II: solid tumors, 1958-1987. Radiat Res 1994;137:S17-67.

6 United National Scientific Committee on the Effects of Atomic Radiation (UNSCEAR). Sources and effects of ionizing radiation. 1993 Report to the General Assembly, with scientific annexes. New York: United Nations, 1993.

7 Thomas G, Karaoglou A, Williams ED, eds. Radiation and thyroid cancer. Singapore: World Scientific, 1999.

- This book describes, inter alia, studies of thyroid cancer among the Japanese A-bomb survivors and those exposed to fallout from Chernobyl and weapons tests.

8 de Vathaire F, Le Vu B, Challeton-de Vathaire C. Thyroid cancer in French Polynesia between 1985 and 1995: influence of atmospheric nuclear bomb tests performed at Mururoa and Fangataufa between 1966 and 1974. Cancer Causes Control 2000;11:59-63.

9 Kerber RA, Till JE, Simon SL, et al. A cohort study of thyroid disease in relation to fallout from nuclear weapons testing. JAMA 1993;270:2076-82.

10 Gilbert ES, Tarone R, Bouville A, et al. Thyroid cancer rates and ${ }^{131}$ doses from Nevada atmospheric nuclear bomb tests. J Natl Cancer Inst 1998;90:1654-60.

11 Stevens W, Thomas DC, Lyon JL, et al. Leukemia in Utah and radioactive fallout from the Nevada test site. A case control study. JAMA 1990;264:585-91.

12 Institute of Medicine. The five series study: mortality of military participants in US nuclear weapons tests. Washington, DC: National Academy Press, 2000.

13 United National Scientific Committee on the Effects of Atomic Radiation (UNSCEAR). Sources and effects of ionizing radiation. 1994 Report to the General Assembly, with scientific annexes. New York: United Nations, 1994.
14 Darby SC, Olsen JH, Doll R, et al. Trends in childhood leukaemia in the Nordic countries in relation to fallout from atmospheric nuclear weapons testing. BMJ 1993;304:1005-9.

15 Karaoglou A, Chadwick KH. Health consequences of Chernobyl and other radiation accidents. Radiat Environ Biophys 1998;37:1-9.

- This paper summarises projects in the former Soviet Union that the European Commission has been supporting.

16 Astakova LN, Anspaugh LR, Beebe GW, et al. Chernobyl-related thyroid cancer in children of Belarus: a case-control study. Radiat Res 1998;150:349-56.

17 Parkin DM, Clayton D, Black RJ, et al. Childhood leukaemia in Europe after Chernobyl: five year follow-up. Br J Cancer 1996;73:1006-12.

18 Petridou E, Trichopoulos D, Dessypris N, et al. Infant leukaemia after in utero exposure to radiation from Chernobyl. Nature 1996;382:352-3.

19 Muirhead CR. Childhood cancer and nuclear installations: a review. Nucl Energy 1998;37:371-9.

20 Hatch MC, Beyea J, Nieves JW, et al. Cancer near the Three Mile Island nuclear plant: radiation emissions. Am J Epidemio 1990;132:397-412.

21 Talbott EO, Youk AO, McHugh KP, et al. Mortality among the residents of the Three Mile Island accident area: 1979-1992. Environ Hith Perspect 2000;108:545-52.

22 McGeoghegan D, Binks K. Mortality and cancer registration experience of the Sellafield employees known to have been involved in the 1957 Windscale accident. J Radiol Prot 2000;20:261-74.

23 Crick MJ, Linsley GS. An assessment of the radiological impact of the Windscale reactor file, October 1957. Int J Radiat Biol 1984;46:479-506.

24 Davis S, Kopecky KJ, Hamilton TE, et al. Hanford thyroid disease study. Draft final report, 1999. http://www.fhcrc.org/science/phs/htds/

25 Kossenko MM, Degteva MO, Vyushkova OV, et al. Issues in the comparison of risk estimates for the population in the Techa Rive region and atomic bomb survivors. Radiat Res 1997;148:54-63.

26 National Radiological Proctection Board. Emergency reference levels of dose for early countermeasures to protect the public: recommendations for the practical application of the board's statement. Doc NRPB 1990;1(4):5-33.

website extra

Additional references appear on the Occupational and Environmental Medicine website

www.occenvmed.com

\section{QUESTIONS (See answers on page 431)}

(1) Which of the following statements about epidemiological studies are true?

(a) It is important to assess statistical power after completing a study

(b) In a case-control study, it is helpful to select cases and controls from different populations, in order that the findings can be generalised

(c) Correlation studies allow the impact of individual exposures to be assessed

(d) Random errors in individual dose estimates tend to bias trends in risk with dose towards zero

(e) All other things being equal, studies with a wide range of doses will tend to be more informative about radiation risks than studies with a narrow dose range

(2) Which of the following statements about the Life Span Study of Japanese A-bomb survivors are true?

(a) Information has been collected on both mortality and cancer incidence

(b) Almost all of the survivors had died by the early 1990s

(c) Most of the excess cancers have been cases of leukaemia

(d) The relative increase in cancer risk is greater for those irradiated in childhood than for those irradiated at older ages 
(e) For all types of cancer, the risk increases linearly with increasing radiation dose

(3) Which of the following statements about studies of nuclear weapons testing are true?

(a) Many of these studies have looked at leukaemia

(b) Most of these studies have used a case-control design

(c) The Marshall Islanders received relatively high radiation doses to the thyroid as a consequence of weapons testing

(d) Leukaemia rates among people exposed to fallout from the Nevada test site are inconsistent with existing risk estimates

(e) Epidemiological studies have been conducted over several decades of groups in the former Soviet Union exposed to weapons fallout

(4) Which of the following statements about the effects of the Chernobyl accident are true?

(a) The main health impact identified to date is an increased risk of thyroid cancer and leukaemia in contaminated areas of the former Soviet Union

(b) Most of the studies of thyroid cancer performed to date have used a case-control design

(c) Uncertainty in individual estimates of radiation doses has limited the quantification of thyroid cancer risks

(d) Radiation doses to people living outside the former Soviet Union were generally much lower than those to people resident in Belarus, Ukraine, and western Russia at the time of the accident

(e) Most of the workers involved in the clean up of the Chernobyl plant have since died from cancer

(6) Which of the following statements about other incidents at nuclear plants are true?

(a) Radiation doses to people living near Three Mile Island at the time of the 1979 accident are comparable in size to the doses to residents near Chernobyl at the time of the 1986 accident

(b) Raised risks of thyroid cancer have been identified following releases of radioactive iodine from the Hanford nuclear site in the USA during the 1940s and 1950s

(c) The population living near the Techa River in the southern Urals in Russia received substantial internal and external radiation exposures over many years as a consequence of operations at a nearby nuclear site

(d) Biological methods may be of some value in estimating exposures resulting from past incidents in the former Soviet Union

(e) All studies of cancer following nuclear incidents are equally informative

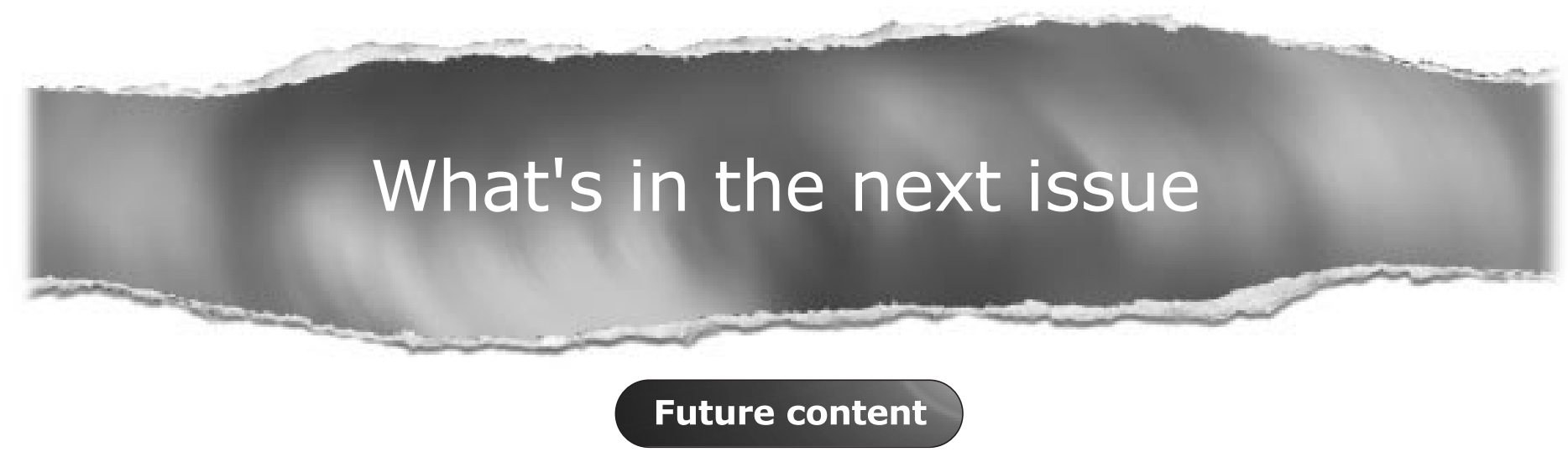

See which articles have just been accepted for publication and preview the table of contents for the next issue a month before it is published

www.occenvmed.com 